\title{
EDITORIAL
}

\section{Neurociencia cognitiva social: el abordaje de la sociedad desde el funcionamiento neurocognitivo}

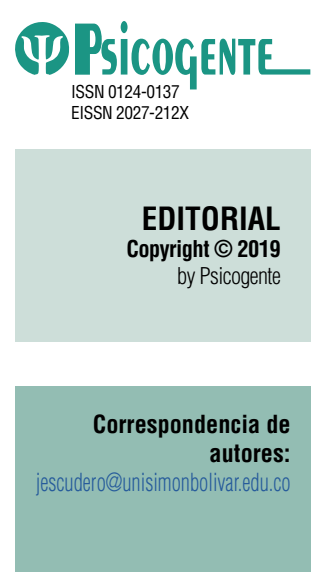

https://doi.org/10.17081/ psico.22.42.354
Johana Margarita Escudero-Cabarcas (ID

PhD Psicología con Orientación en Neurociencia Cognitiva

Grupo sinapsis educativa y social

Universidad Simón Bolívar

La comprensión de los estados emocionales, de las actitudes, de la forma como hombres y mujeres percibimos, comprendemos al otro, de lo que nos motiva, nos moviliza a la acción, nos conecta con los seres humanos y con el mundo que nos rodea, está enmarcada en lo que se conoce en la actualidad como: Neurociencia Cognitiva Social. Esta es entendida como el estudio del procesamiento de la información social y el uso que se le da a esta información, partiendo tanto de las condiciones del funcionamiento cerebral, como de la interacción social (Pineda, 2000; Pineda-Alhucema, 2016).

Lo que nos lleva a considerar la interacción constante de la tríada MenteCerebro y Sociedad en una conjunción que va más allá de los lineamientos particulares que el estudio de cada componente ha gestado a lo largo del desarrollo científico de su área de acción teórica y propende por la generación de nuevas formas de ver al ser humano desde la integralidad de los aspectos que la comprensión del Cerebro, su anatomía y su funcionamiento; la Mente y sus manifestaciones desde lo normal y lo patológico, y la Sociedad como causa y producto de las interacciones gestan alrededor del ser humano como sujeto de su estudio.

Es así como en la actualidad la neurociencia cognitiva social nos ofrece un gran campo de acción, llevándonos al conocimiento de aspectos tales como: La Percepción social, la cual implica la Percepción de rostros y cuerpos, el movimiento, la comprensión de las acciones, Teoría de la Mente, Empatía. Por otro lado, la Comprensión del sí mismo que conlleva aspectos como el reconocimiento de sí mismo, reflexión sobre sí mismo. Así como la Autorregulación social que en sus componentes lleva al estudio de autorregulación intencional, autorregulación no intencional. A su vez la Interacción con el mundo social la cual nos invita a estudiar las neuronas espejo e imitación, actitudes y prejuicio, aceptación y rechazo social, toma de decisiones sociales. 
(Lieberman, 2007; Fiske \& Taylor, 2007), entre otras tantas temáticas que aún están por explorar y que nos permitirían seguir comprendiendo al ser humano desde su composición neurobiológica en interacción constante con el medio.

Vista de esta manera, podríamos considerar a la Neurociencia cognitiva social como una disciplina en construcción que además de ofrecernos una mirada interdisciplinar del ser humano, nos invita desde la psicología a integrar los saberes en procesos de conducta y a retomar a las neurociencias y sus explicaciones de la composición y funcionamiento del cerebro para comprender cómo el medio social influye en las estructuras cerebrales y cómo estas a su vez nos llevan a ver el mundo y a interactuar en él tal y como lo hacemos (Pineda-Alhucema, Aristizábal, Escudero-Cabarcas, Acosta-López \& Vélez, 2018; Guerrero-Martelo, Galván, Vásquez De la hoz, Lázaro \& Morales, 2015).

\section{REFERENCIAS}

Fiske, S., \& Taylor, S. (2007). Social Cognition, from Brains to Culture. Georgia, USA: McGraw-Hill

Guerrero-Martelo, M., Galván, G., Vásquez De la hoz, F., Lázaro, G., \& Morales, D. (2015). Relación entre autoestima y autonomía funcional en función del apoyo social en adultos institucionalizados de la tercera edad. Psicogente, 18(34), 303-310. http://publicaciones.unisimonbolivar.edu.co/rdigital/ojs/index.php/ psicogente/article/view/506/0

Lieberman, M. D. (2007). Social cognitive neuroscience: a review of core processes. Annu RevPsychol, 58, 259-289. https://www.ncbi.nlm.nih.gov/pubmed/17002553

Pineda, D. (2000). La función ejecutiva y sus trastornos. Rev. Neurol, 30(8), 764-768. https://doi.org/10.33588/rn.3008.99646

Pineda-Alhucema, W. (2016). La Teoría de la Mente desde el Marco de la Neurociencia Cognitiva Social. Psicogente, 19(35). http://revistas.unisimon.edu.co/ index.php/psicogente/article/view/1381

Pineda-Alhucema, W., Aristizábal, E., Escudero-Cabarcas, J., Acosta-López, J.E., \& Vélez, J.I. (2018). Executive Function and Theory of Mind in Children with ADHD: a Systematic Review. Neuropsychology Review, 28(3), 341-358. https://doi. org/10.1007/s11065-018-9381-9 